\begin{tabular}{|l|l|l|}
\hline \multicolumn{2}{|c|}{ PublisherInfo } \\
\hline \hline PublisherName & $:$ & BioMed Central \\
\hline \hline PublisherLocation & $:$ & London \\
\hline \hline PublisherImprintName & $:$ & BioMed Central \\
\hline \hline
\end{tabular}

Improved oligonucleotides for microarrays

\begin{tabular}{|l|l|l||}
\hline \multicolumn{2}{|c|}{ ArticleInfo } \\
\hline \hline ArticleID & $:$ & 3566 \\
\hline \hline ArticleDOI & $:$ & $10.1186 /$ gb-2000-1-1-reports032 \\
\hline \hline ArticleCitationID & $:$ & reports032 \\
\hline \hline ArticleSequenceNumber & $:$ & 57 \\
\hline \hline ArticleCategory & $:$ & Paper report \\
\hline \hline ArticleFirstPage & $:$ & 1 \\
\hline \hline ArticleLastPage & $:$ & 4 \\
\hline \hline & & RegistrationDate : 2000-3-2 \\
ArticleHistory & $:$ & Received \\
& $: 2000-3-2$ \\
\hline \hline ArticleCopyright & $:$ & BioMed Central Ltd2000 \\
\hline \hline ArticleGrants & $:$ & \\
\hline \hline \hline ArticleContext & $:$ & 130591111 \\
\hline \hline
\end{tabular}




\section{Elaine Mardis}

\section{Abstract}

A novel method for the synthesis of 5 '-attached oligonucleotides that eliminates truncated sequences may improve microarray construction.

\section{Significance and context}

Synthesis of oligonucleotides attached to solid supports is crucial in the production of DNA microarrays. Synthesis on the support is hampered by two problems - truncation of synthesized oligonucleotides, and the need for oligonucleotides to be synthesized in the $5^{\prime}$ to $3^{\prime}$ direction, opposite to the conventional direction of DNA synthesis. Although in situ synthesis in the $5^{\prime}$ to $3^{\prime}$ direction is possible, it gives a significantly lower yield than in the conventional $3^{\prime}$ to 5 ', and results in a higher proportion of truncated sequences. Inclusion of truncated products in microarrays is a problem for various reasons; primarily, they contribute to increased background. Eliminating them from the array would decrease this background significantly and should improve error rates. Kwiatkowski et al. describe a method by which oligonucleotides can be inverted in situ after synthesis in the $3^{\prime}$ to $5^{\prime}$ direction. The $5^{\prime}$ end of the oligonucleotide terminates with a functionality that enables the covalent attachment of that end to the solid support while the $3^{\prime}$ end remains attached. Subsequent treatment releases the $3^{\prime}$ end, providing a free $3^{\prime}-\mathrm{OH}$, which can be used in a variety of assays such as minisequencing and pyrosequencing and allows the release of most truncated synthesis products, as these do not have the $5^{\prime}$ functionality necessary for attachment. The authors use a variety of analytical and functional assays to characterize their method.

\section{Key results}

Under the conditions tested, the authors report that the best yield of inverted oligonucleotides represented about $25 \%$ of the maximum amount of material that could have been synthesized. Analysis of inversion products following in situ synthesis of mixed-length oligonucleotides, which mimic truncated synthesis products, indicated that the inversion process resulted in effective in situ oligonucleotide purification by eliminating truncated products from the solid support. The inverted products were further tested for function in minisequencing and pyrosequencing assays. Both assays indicated that DNA polymerase could add nucleotides to the $3^{\prime}-\mathrm{OH}$ end of an oligonucleotide resulting from inversion. 


\section{Methodological innovations}

There are extensive descriptions of the innovative chemical derivatization of the solid support and of the in situ synthesis protocols which enable the inversion of oligonucleotides. Because "synthesis on planar solid supports results in a limited quantity of product", the authors used 50-70 $\mu \mathrm{m}$ diameter polystyrene beads to obtain sufficient product to monitor the individual steps of the inversion procedure. The 5' functionality that allowed inversion was an added o-chlorophenyl phosphodiester moiety, which in the presence of a condensing agent forms a phosphodiester linkage between the 5 ' end of the oligonucleotide and the solid support. Analysis by capillary electrophoresis of reaction products cleaved from the solid support indicated that two major products were obtained after inversion - a mix of $5^{\prime}$ inverted and non-inverted 19 base oligonucleotides (19mers). The 5' inverted 19mers occupied a physically distinct peak on the chromatogram as a result of the attachment of several triethyleneglycol moieties, added to facilitate cleavage of the oligonucleotides from the solid support and to distinguish inverted oligonucleotides from truncated products.

\section{Conclusions}

According to the authors, this method is suitable for the production of oligonucleotide arrays and is compatible with all existing methods for in situ oligonucleotide synthesis. In contrast to other methods for in situ synthesis, incomplete sequences are removed by this method.

\section{Reporter's comments}

This is a preliminary description of a potentially important new method that tackles several of the problems inherent in oligonucleotide synthesis in situ on solid supports. The authors chose polystyrene beads for these preliminary experiments because of the ease of monitoring and analyzing the synthetic stages. The next step in substantiating the method will be to reproduce and characterize the results on more conventional microarray supports such as glass slides. It will also be interesting to determine the range of oligonucleotide lengths that undergo the inversion process with reasonable success, and how inversion yield is influenced by oligomer length.

\section{Table of links}

Nucleic Acids Research 


\section{References}

1. Kwiatkowski M, Fredriksson S, Isaksson A, Nilsson M, Landegren U: Inversion of in situ synthesized oligonucleotides: improved reagentsfor hybridization and primer extension in DNA microarrays. Nucleic Acids Res. 1999, 27: 4710-4714. 0305-1048

This PDF file was created after publication. 